\title{
Distributed Power Dispatch via Bifurcation Control
}

\author{
Pirathayini Srikantha and Deepa Kundur \\ The Edward S. Rogers Sr. Department of Electrical and Computer Engineering \\ University of Toronto \\ Email: \{pirathayini.srikantha, dkundur\}@ece.utoronto.ca
}

\begin{abstract}
Distributed generators (DGs) are becoming widely deployed in today's power grid. These energy sources are typically sustainable and cost-effective but are highly variable based on local environmental conditions. This variability poses many challenges to the practical deployment of DGs in the grid. In this work, a novel distributed power dispatch strategy based on bifurcation control that capitalizes upon the recent cyber enablement of the grid is proposed to effectively manage DGs implemented at the distribution substation level of the power grid. This scheme enables every participating DG to locally compute its dispatch strategy based on simple cyber signals broadcasted by the utility. Results indicate that the DGs are able to rapidly converge to the optimal economical power dispatch with significantly less concentrated computational effort and communication overhead.
\end{abstract}

\section{INTRODUCTION}

Distributed generators (DGs) are sustainable energy sources that are becoming highly prevalent in today's grid - especially in the residential sector. Examples of DGs include PhotoVoltaics (PVs) and wind turbines. The main grid mostly contains non-distributed synchronous generation sources that have predictable generation capacities but are geographically dispersed. In the residential sector, DGs are particularly advantageous as these are deployed at close proximity of the demand (i.e. roof-top solar panels) enabling higher efficiency due to reduced transmission line losses. Despite the many advantages of DGs, practical deployment is challenging as generation capacities of DGs are highly variable. In order to maintain stability in the grid, generation must balance the demand accurately. Since, the generation capacities of DGs are influenced by external conditions such as the weather, highly accurate predictive models are required to plan the dispatch of distributed generation. In the literature, centralized optimization techniques are employed to compute power dispatch [3], [20] which can become intractable as the number of DGs in the system increases.

The recent cyber enablement of the grid allows communication between various components in the power system. In this work, a novel distributed dispatch strategy based on bifurcation control is proposed that capitalizes on the extensive communication infrastructure present in the grid. Bifurcation is defined to be the study of how the equilibrium or fixed points of a non-linear system can change when certain parameters in the system are varied. In engineering systems, this is typically used to conduct stability analysis in the presence of perturbations to certain parameters in the system [14], [6]. Stability analysis is important for understanding and imposing safety margins in engineering applications. In power systems, works such as [7], [8] have used bifurcation methodologies to conduct this type of analysis. Bifurcation has also been used in many control applications belonging to various engineering disciplines such as aerospace engineering (jet engine compression), power systems (voltage regulation in power system), mechanical engineering (thermal control) and biomechanics (cardiac cycles) [5]. These works use bifurcation control to change the operating points of the system to more desirable ones under various circumstances. For instance, in the work of [18], bifurcation is used to control nonlinear dynamical systems so that performance and stability trade-offs are balanced. Bifurcation control proposed in [16] limits oscillatory amplitudes in the system. [1] implements bifurcation control that takes into account hard constraints due to limitations in various components of non-linear feedback systems. [9] shows that bifurcation control can be integrated into the design of a global controller that utilizes qualitative and quantitative knowledge in the system to devise optimal control strategies.

As per the authors' knowledge, bifurcation techniques have not been applied for control in distributed systems in engineering applications. In developmental biology, bifurcation is used to distributively control and dictate the function of cells by adjusting the concentration of morphogens present at various regions in an embryo [19]. In this work, the bifurcation control paradigm used in developmental biology is applied to the power system to distributively control the amount of power dispatched by DGs so that the demand in the system is balanced by the dispatched generation. The utility computes and broadcasts signals that reflect the degree of balance between aggregate generation and demand in the system to DGs. These signals can alter the equilibrium points of every participating DG inducing change in the dispatch states of the DGs. This is repeated until the DGs converge to the optimal dispatch that balances the overall demand in the system. This scheme completely decouples the DGs from the main utility enabling optimal dispatch under realistic generation conditions.

The remainder of this paper is organized as follows. The system model used in this paper for generation dispatch is presented in Section II. The proposed distributed dispatch algorithm based on bifurcation control is detailed in Section III. This scheme is implemented in a realistic environment and the corresponding results are listed in Section IV. Finally, the paper is concluded in Section V. 


\section{System MODEL}

The dispatch problem is formulated at the distribution substation level for a residential community interspersed with DGs and is illustrated in Figure 1. The synchronous generation plant is the main supplier of power in the main grid. This power is transported via transmission lines to the distribution substation. In order to reduce transmission losses, the transmission lines operate at high voltages. The distribution substation steps down the voltage of the power supplied by the transmission lines and transfers power to a pole-top transformer. This transformer serves homes and commercial entities in the residential sector via underground cables. These homes may or may not be equipped with a DG source. A long range wireless transmitter is mounted on the pole-top transformer which broadcasts signals from the utility. The goal of the proposed scheme is to reduce the dependence of residential communities on synchronous generators as these can be expensive, subject to inefficiencies due to transmission losses and possibly be associated with significant carbon footprint.

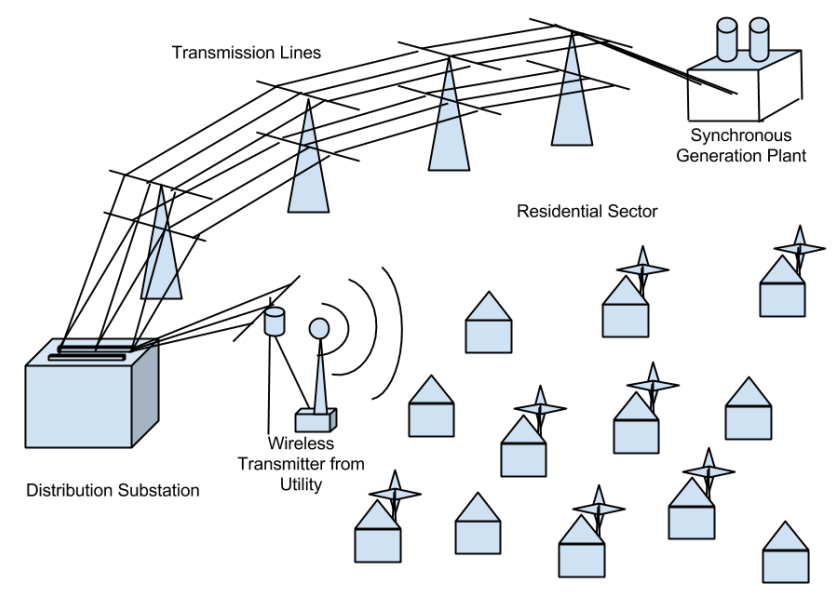

Fig. 1. Power system diagram at the distribution substation level

\section{A. Assumptions}

Following is a list of all the assumptions made in the formulation of the DG dispatch problem.

1) Utility contains a wireless transmitter to broadcast cyber signals over the cellular medium;

2) Each DG is fitted with a cyber-physical device that can be programmed with the logic for computing local dispatch. The cyber element is capable of receiving cellular broadcasts. The actuating component controls the power dispatched to the system;

3) System is connected to the main grid containing negative spinning reserves, synchronized generation resources and storage systems;

4) Utility can implicitly measure the power supplied or absorbed by generation in the main grid;

5) Cost of power dispatched by DGs is less than power purchased from the main grid;
6) Demand and generation capacities of DGs remain constant for at least 5 minutes.

The first and second assumptions are based on the cyberenablement of the power grid. The third assumption is necessary to ensure that the system remains in stability by absorbing or injecting excessive or deficit power in the system while the DGs distributively converge to the optimal dispatch solution. The fourth assumption is feasible as the utility will have access to the power generation data in the main grid. The fifth assumption is necessary to tap onto the generation potential of DGs rather than the synchronous generators. The sixth assumption is valid as this interval is less than the day-ahead dispatch performed in today's grid [11] and therefore less prone to errors in predictive models.

\section{B. Original Problem}

The original problem for economic power dispatch is formulated as $P_{P}$

$$
\begin{aligned}
& \left(\mathbf{P}_{P}\right) \min _{x} f_{o}(x) \\
& \text { subject to } \sum_{i=1}^{n} x_{i}-\sum_{j=1}^{p} L_{j}=0 \\
& 0 \leq x_{i} \leq c_{i} \quad \forall \mathrm{i}=1 \ldots \mathrm{n}
\end{aligned}
$$

where $x \in R^{n}$ is the dispatch vector $(\mathrm{kW})$ of $n$ DGs, $f_{o}(x)$ is the generation cost function $(\$), \sum_{j}^{p} L_{j}$ is the aggregate power demand $(\mathrm{kW})$ in the system from $p$ loads and $c_{i}$ is the generation capacity $(\mathrm{kW})$ of each DG. The coupling constraint $\sum_{i}^{n} x_{i}-\sum_{j}^{p} L_{j}=0$ ensures that the total supply meets the aggregate demand in the system. The inequality constraints specify the valid dispatch range for every DG. This centralized problem contains $n$ variables and $2 n+1$ constraints. As the number of DGs increases in the system, the computational overhead that results when $P_{P}$ is solved in a central manner similar to works in [3], [20] and [17] can be excessive. In addition to this, the utility will require knowledge of local conditions from DGs to compute $c_{i}$ which will extensively increase communication overhead. On the other hand, distributed solutions in the literature like that in [10] and [4] may not be suitable for real-time dispatch due to slow convergence characteristics.

\section{Distributed Dispatch SCHEME}

In this section, the proposed distributed dispatch scheme is presented in detail. The distributed dispatch scheme allows every DG to locally compute its optimal dispatch in an iterative manner based on signals transmitted by the utility. According to Assumption 6, power demand and generation is constant over every 5 minute interval. Each interval will be referred to as the dispatch interval.

\section{A. Utility}

The utility will broadcast a signal $u_{t}$ to DGs at every signalling iteration. The signal contains information about the degree to which generation meets demand in the system and is calculated according to Equation 2: 


$$
u_{t}=\frac{\sum_{j=1}^{n} x_{j}}{\sum_{k=1}^{p} L_{k}}
$$

where $u_{t}$ is the signal computed at the $t^{t h}$ signalling iteration. Signals are transmitted every $d$ seconds. As the information content of $u_{t}$ is simple and applies to all DGs in general, communication occurs only in the downlink in a broadcast fashion resulting in minimal overhead.

\section{B. Distributed Generators}

Power dispatch of each DG evolves as a non-linear dynamical system. The DGs are each assigned a parametrized dispatch vector field function. The general form of this function for the $i^{t h}$ DG is listed in Equation 3:

$$
\dot{x}_{i}\left(s_{i}\right)=\alpha C_{i}\left(x_{i}-s_{i}\right)\left(\left(c_{i}-s_{i}\right)^{2}-\left(x_{i}-s_{i}\right)^{2}\right)
$$

where $\alpha$ is a cost adjustment factor, $s_{i}$ is the parameter adjusted by the DG based on signal $u_{t}$ transmitted by the utility. The vector field function can be different for every DG based on individual generation costs $C_{i}$ and capacities $c_{i}$. Although Equation 3 resembles the normal function for transcritical bifurcation, this is not the case. Figure 2 illustrates an instance of the dispatch vector field diagram when both $c_{i}=4 \mathrm{~kW}$ and $s_{i}=2$ are fixed. At steady state, DGs will operate at an equilibrium point. The signal transmitted by the utility serves as an external 'disturbance' to the dispatch vector field function which can possibly result in a change to the equilibrium points of the DGs. If a change in the current equilibrium point of the DG occurs, then the DG will begin to evolve towards the new stationary point.

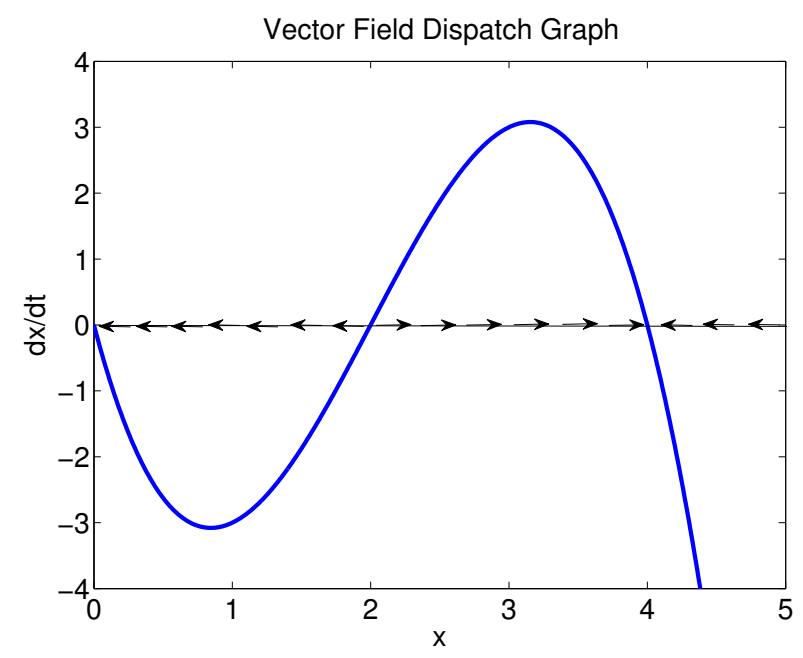

Fig. 2. Instance of gradient function for $c_{i}=4$

Figure 3 illustrates the bifurcation diagram of the normal function in Equation 3. This diagram contains all stationary points possible for various values of the parameter $s_{i}$. When $s_{i}<c_{i}$, three equilibrium points exist out which two are stable. The unstable equilibrium point occurs at $\bar{x}_{i}=s_{i}$. The

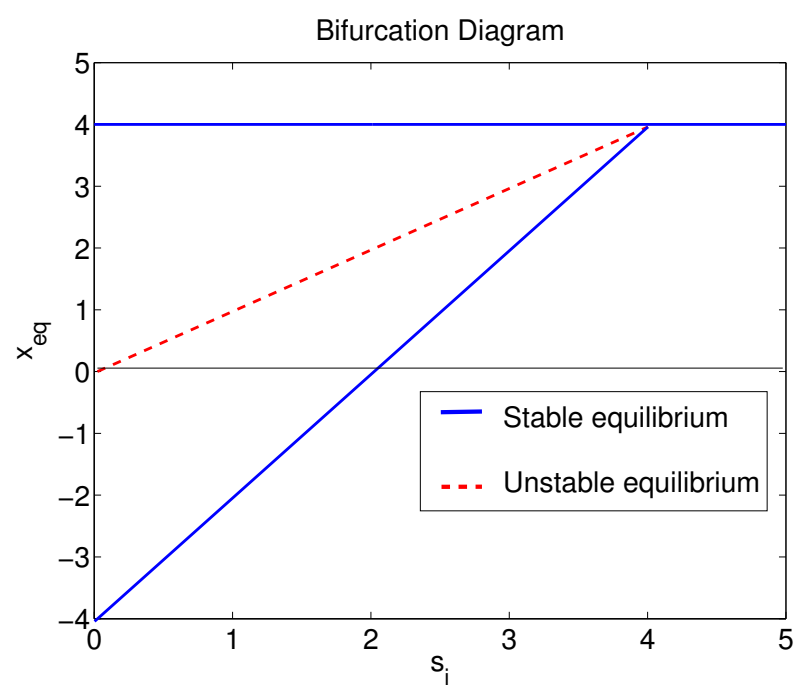

Fig. 3. Bifurcation diagram for normal function in Equation 3 for $c_{i}=4$

stable equilibrium points occur at $\bar{x}_{i}=c_{i}$ and $\bar{x}_{i}=2 s_{i}-c_{i}$. Since the DGs can only dispatch positive power, negative equilibrium points $\bar{x}_{i}<0$ are not considered. When $s_{i} \geq c_{i}$, only one equilibrium point exists and this is $\bar{x}_{i}=c_{i}$. It is clear from Figure 3 that all equilibrium points exist between 0 and $c_{i}$.

The signal transmitted by the utility dictates how the equilibrium points are shifted. The mapping of $u_{t}$ to the parameter $s_{i}$ is performed by every participating DG as follows. When aggregate generation is lesser than demand (i.e. $0<u_{t}<1$ ), DGs should increase generation dispatch cautiously as a sudden increase can result in oscillations if there are many DGs in the system. A DG will evaluate whether its current dispatch is less than the recent value of $s_{i}$. If so, it will set $s_{i}$ to $x_{i}-\epsilon$. This way, the DG can evolve in a positive direction and increase its dispatch by starting off slowly as dictated by the vector field function. When generation is more than demand (i.e. $1<u_{t}$ ), then it is necessary to decrease dispatch in a cautious manner. Hence when a DG evaluates its current dispatch to be greater than the recent value of $s_{i}, s_{i}$ is set to $x_{i}+\epsilon$. This way, the dispatch will now move away from the unstable equilibrium point $s_{i}$ in a negative direction. If $u_{t}=1$ then the DG will fix its current dispatch. Table I provides a summary of the proposed distributed dispatch algorithm.

\section{EVALUATION}

In this section, the proposed distributed dispatch system is implemented in MATLAB using realistic generation and demand models. First a discussion of the models used in the implementation is provided. Then, results obtained from applying the dispatch strategy in the simulation environment is presented.

\section{A. Modelling of DGs}

In this work, PVs and wind turbines are the only types of DGs considered. 


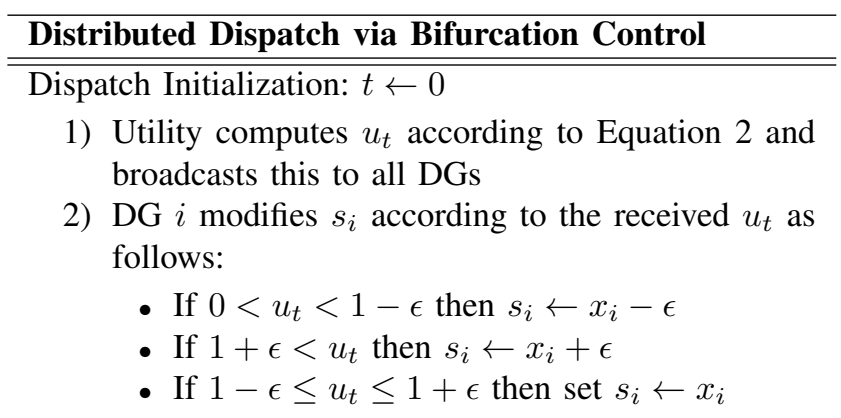

3) DG begins the dispatch evolution process according to Equation 3

4) After $d$ seconds, the utility initiates iteration $t \leftarrow$ $t+1$ by returning to step 1

\section{TABLE I}

DISTRIBUTED DISPATCH ALGORITHM FOR A SINGLE CYCLE

Generation capacity of PVs depends on amount of solar irradiance striking the solar panels. Data obtained from [13] containing hourly solar power generation data by panels with $3.08 \mathrm{~kW}$ AC rating in the region of Toronto on a randomly selected day is used in this work to represent the power generation capacity of PVs over a day. It is assumed that the power capacity of PVs remains constant during an hour.

Generation capacity of a wind turbine is characterized by four parameters and these are cut-in speed, rated speed, cutoff speed and rated power output. Power generated by a wind turbine for wind speeds $v$ in between the cut-in and rated speeds is represented by Equation 4 .

$$
P_{\text {wind }}=\frac{1}{2} A \rho \theta v^{3}
$$

where $A$ is the area of wind that passes through the rotors of the turbine with diameter $D, \rho$ is the air density and $\theta$ is the power output efficiency of the turbine. Wind turbines considered in this work are assumed to have $50 \mathrm{~kW}$ power rating, $25 \mathrm{~m}$ height, $13 \mathrm{~m}$ rotor diameter and 0.4 efficiency [15]. Wind speeds can be modelled using the Weibull probability density model for 5 minute forecasts [12]. In this work, wind speed is assumed to be characterized by the Weibull probability density function with a shape factor of 1.94 and a scale factor of 4.48 .

\section{B. Demand Modelling}

Demand from the residential sector can differ due several factors such as seasonal changes, properties of households in the region and penetration rate of electric appliances in the households. A method combining all these factors to model demand in the residential sector has been proposed in [2]. This method groups homes into four classes. The power demand from homes belonging to each of these classes is modelled as a continuous time Markov Process. States and the rate matrices for all four classes have been constructed from actual measurement data obtained from a testbed of sample of homes. In this work, homes belonging to Class 1 with a peak load of $3902 \mathrm{~W}$ and base load of $142 \mathrm{~W}$ are considered. The region considered in this work is assumed to contain 10 Class 1 homes.

\section{Results}

The dispatch strategy proposed in this work is implemented in the simulation environment containing 10 homes, 4 wind turbines and 6 solar panels for three dispatch cycles. Results are illustrated in Figure 4. These results illustrate how the DGs are able to adapt to changes in the demands and generation capacities that can occur across dispatch cycles.

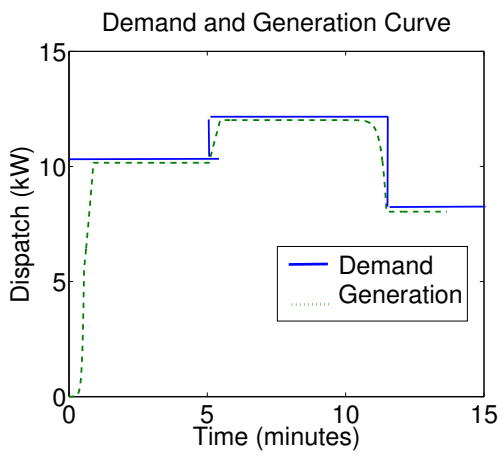

Fig. 4. Results

It is clear that all the DGs are able to quickly ramp up dispatch to meet the aggregate demand in the system in the first dispatch interval. In the second dispatch interval, the aggregate demand increases. The DGs are also able to distributively compute the optimal dispatch and automatically adapt to the increase. In the third interval, the overall demand in the system drops to lower value. The DGs cautiously reduce individual dispatch to finally converge to the aggregate demand. The rate of dispatch reduction is initially slow and then rapid as illustrated by the plot in Figure 3. Hence, these results indicate that bifurcation control can be used effectively dispatch power supply of DGs in a distributed manner. In comparison to stateof-the-art centralized strategies, our strategy performs well as it entails low communication costs and information overhead, and is not subject to forecasting errors. Since the proposed strategy is distributed, privacy and security in the system is enhanced as disruptions from single point of failure will not occur.

\section{CONCLUSION}

In this work, it is shown that it is possible to use principles from bifurcation control to manage distributed systems. A novel distributed dispatch strategy has been proposed that enables DGs to converge very rapidly to the optimal dispatch vector in a real-time manner while entailing low communication and information overhead. The flexibility, efficiency, scalability and reduced computational complexities render it ideal for deployment in practical systems containing a large and diverse number of DGs. As future work, it will be interesting to extend the dispatch strategy proposed in this work across systems served by multiple distribution substations which will introduce new constraints at the transmission level. 


\section{REFERENCES}

[1] Diego M. Alonso, Daniel W. Berns, Eduardo E. Paolini, and Jorge L. Moiola. Bifurcation control in feedback systems. Bifurcation Control, page 205228, 2003.

[2] Omid Ardakanian, Srinivasan Keshav, and Catherine Rosenberg. Markovian models for home electricity consumption. in proceedings of the ACM Sigcomm 2011 workshop on Energy and IT: from Green Networking to Smarter Systems, pages 31-36, 2011.

[3] M. Renu Avinaash, G. Ravi Kumar, K. Anjaneya Bhargav andT. Srikanth Prabhu, and D. IndraSena Redd. Simulated annealing approach to solution of multi-objective optimal economic dispatch. International Conference on Intelligent Systems and Control, pages 1-6, 2013.

[4] Giulio Binetti, Mohammed Abouheaf, Frank Lewis, David Naso, Ali Davoudi, and Biagio Turchiano. Distributed solution for the economic dispatch problem. 21st Mediterranean Conference on Control and Automation, pages 243-250, 2013.

[5] G. Chen, J. L. Moiola, and Wang. Bifurcation control: Theories, methods, and applications. International Journal on Bifurcation Chaos, 10(3):511548, 2000.

[6] Iooss G. and Joseph D. D. Elementary stability and bifurcation theory. New York:Springer, 1980.

[7] Abed E. H. Bifurcation-theoretic issues in the control of voltage collapse. In Proc. IMA Workshop on Systems and Control Theory for Power Sys- tems, 1995.

[8] Nayfeh A. H., Harb A. M., and Chin C. M. Bifurcation in power system model. International Journal on Bifurcation Chaos, 6:497512, 1996.

[9] Joseph Leung, David J. Hill, Yixin Ni, and Ron Hui. Global power system control using a unified power flow controller. PSCC, 14:1-6, 2002.

[10] Hao Liang, Bong Jun Choi, Atef Abdrabou, Weihua Zhuang, and Xuemin (Sherman) Shen. Decentralized economic dispatch in microgrids via heterogeneous wireless networks. IEEE Journal on Selected Areas in Communications, pages 1061 - 1074, 2012.

[11] Hydro One. The electricity sector, October 2013.

[12] Kostas Philippopoulos and Despina Deligiorgi. Statistical simulation of wind speed in athens,greece based on weibull and arma models. International Journal of Energy and Environment, 3(4):151-158, 2009.

[13] PVWatts. Ac energy and cost savings, October 2013.

[14] Seydel R. Practical bifurcation and stability analysis - from equilibrium to chaos. Berlin:Springer, 1994.

[15] Wind Energy Resources. $50 \mathrm{kw}$ wind turbine generator, October 2013.

[16] A. Tesi, E. H. Abed, Genesio R., and Wang H. O. Harmonic balance analysis of period-doubling bifurcations with implications for control of nonlinear dynamics. Automatica, page 12551271, 1996.

[17] Arun Kumar Uchchkotiya, Rameshwar Singh, and A.S Trivedi. Evolutionary techniques of gams used in optimization of economic load dispatch in power system. International Journal of Engineering Research and Technology, 2:2359 - 2367, 2013.

[18] Hua O. Wang and Dong S. Chen. Emerging directions in bifurcation control. Bifurcation Control, page 229248, 2003.

[19] Ortrud Wartlick, Anna Kicheva, and Marcos Gonzalez-Gaitan. Morphogen gradient formation. Cold Spring Harbor Perspectives Biology, $1(3), 2009$.

[20] Jianxin Xu. Optimization of economic load dispatch for a microgrid using evolutionary computation. 37th Annual Conference on IEEE Industrial Electronics Society, pages 3192 - 3197, 2011. 